\title{
Depinning transitions between adsorbate chains coupled by Friedel oscillations
}

\author{
G. Godzik, T. Block, and H. Pfnür \\ Institut für Festkörperphysik, Universität Hannover, Appelstrasse 2, D-30167 Hannover, Germany
}

(Received 9 December 2002; published 31 March 2003)

\begin{abstract}
The low coverage phase transitions of the $p(8 \times 1)$ and the $p(5 \times 1)$ chain structures of the $\mathrm{Sr} / \mathrm{Mo}(112)$ system have been investigated with low-energy electron diffraction (LEED) at constant coverages. It turns out that both structures show similar physics. They first undergo depinning phase transitions at temperatures just below $100 \mathrm{~K}$ into striped domain wall structures. The domain wall lattice shows continuous melting at temperatures between 197 and $218 \mathrm{~K}$. Coupling between the adsorbate chains can be described by the potential created by adsorbate-induced charge density waves involving surface states. Within this model domain wall formation appears as thermal excitation of stacking faults on a discrete lattice. For the $p(5 \times 1)$ structure, domain wall formation energies of approximately 65 and $38 \mathrm{meV}$ have been derived for heavy and light walls, respectively, from a fit to the experimental data of depinning.
\end{abstract}

DOI: 10.1103/PhysRevB.67.125424

PACS number(s): 64.70.Rh, 68.35.Rh

\section{INTRODUCTION}

The formation of linear-chain structures at low coverages is a peculiarity of adsorption of atoms of many electropositive elements on channelled metal surfaces, such as the (112) faces of bcc crystals. ${ }^{1}$ These have a similar structure as the (110) fcc surfaces. A particularly rich variety of chain structures has been found not only for alkaline-earth adsorbates but also for rare-earth adsorbates. This was attributed to the rather large electron density in their valence shells and the moderate dipole moments as compared to those for the alkali electropositive adsorbates. These features are believed to be responsible for a greater weight of the indirect interaction between the adatoms, i.e., the interaction mediated through the substrate electrons, relative to the dipole-dipole repulsion along the channels, so that the formation of chainlike $p(n$ $\times 1)$ structures with integer $n$ between 2 and 8 is favored. ${ }^{1}$ Contrary to the (110) surfaces of $\mathrm{Au}$ or Pt, the Mo(112) surface is not reconstructed. ${ }^{2}$

Apart from the formation of strongly coupled chains, which is a local electronic effect, the coupling mechanism between chains over distances up to eight or even nine lattice constants and the relatively high thermal stability ${ }^{3}$ triggered investigations of adsorbate induced vertical and lateral relaxations in some of these systems. $\mathrm{Li}$ and $\mathrm{Sr}$ on $\mathrm{Mo}(112)$ have been investigated in detail by LEED-IV analysis. ${ }^{4,5}$ Wavelike modulations of the substrate density in the topmost three substrate layers have been found that are induced by the adsorbate. These modulations can only come about by coupling of lattice relaxations and electron density at the Fermi surface. Friedel oscillations, induced by the distortions of the electron density due to the adsorbate, couple with the lattice and cause a lateral interaction potential also between the adsorbate chains. Surface states crossing the Fermi level have indeed been found on the clean surface of $\operatorname{Mo}(112) .{ }^{6}$ Since the electron density of states close to the Fermi level from bulk states is low in the topmost substrate layers, as shown in Ref. 6, the influence of surface states actually dominates. Thus, the periodicity of the chain structure of the adsorbate as well as the adsorbate-induced relaxations in the substrate can be considered as being stabilized by the adsorbate- induced charge density waves in these systems. ${ }^{7-11}$

As a consequence, different adsorbates, e.g., $\mathrm{Li}$ and $\mathrm{Sr}$, cause different periodicities between chains. This shows that the Fermi wave vector can effectively be tuned by the adsorbate and by its concentration. Only the electron density in surface states can react sensitively to various adsorbates on metal surfaces, which demonstrates again the crucial role of surface states in the present context.

In this paper we concentrate on the thermodynamic properties of such strongly anisotropic chain systems formed by adsorbed atoms, in particular on the $\mathrm{Sr} / \mathrm{Mo}(112)$ system at coverages up to 0.2 monolayers (ML). The method of electron diffraction, which is used for this study, directly yields the thermodynamic averages of interest. These would be almost impossible to derive with local probes such as tunneling microscopy for the large unit cells of the $p(8 \times 1)$ and $p(5 \times 1)$ structures, which correspond to saturation coverages of 0.125 and $0.2 \mathrm{ML}$, respectively. The LEED patterns of these structures together with real space models are shown in Fig. 1.

The systems investigated here are particularly interesting for two reasons. First, it is well known that strongly anisotropic systems can undergo two types of phase transitions for $n>2$ : a commensurate-incommensurate transition to a striped soliton phase by the formation of domain walls and a second transition of continuous melting of these solitons by unbinding of pairs of kinks. ${ }^{12}$ (Here $n$ is a general commensurate one-dimensional periodicity in units of the substrate lattice constant). Second, in the system presented here, $\mathrm{Sr} /$ $\mathrm{Mo}(112)$, simple chains of $\mathrm{Sr}$ atoms are strongly chemisorbed to the Mo substrate, whereas the distance is determined by the lateral interactions just described, which are comparatively weak. Therefore, domain walls will only be created by discrete variations of chain distance. In addition, the proposed coupling mechanism between chains-a mixture of dipole-dipole repulsion and an oscillatory part due to the charge density waves ${ }^{5}$ - changes the energetics compared with standard elastic media mostly dealt with theoretically.

\section{EXPERIMENT}

To perform the experiments, we used the UHV system and the experimental setup described earlier. ${ }^{3,13,14}$ Briefly, a 
a)
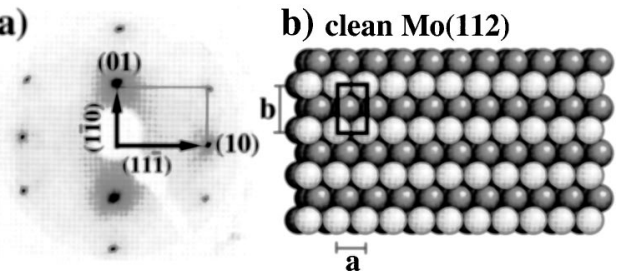

c)

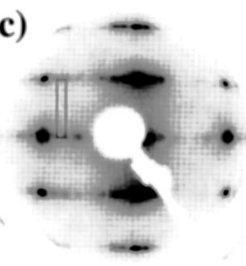

e)

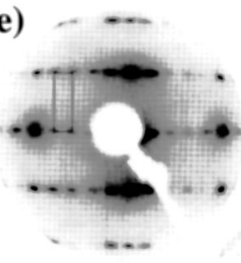

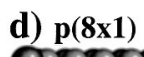

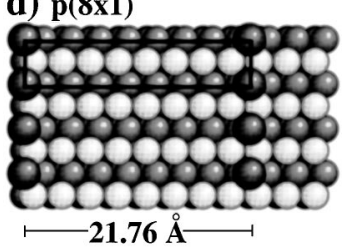

f) $\mathrm{p}(5 \times 1)$

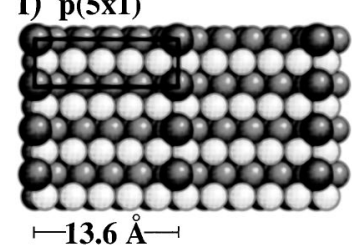

FIG. 1. Reciprocal and real-space images of the clean Mo(112) surface and of the Sr-induced low-coverage commensurate structures $p(8 \times 1)$ and $p(5 \times 1)$.

standard backview LEED instrument equipped with a slow scan charged-coupled device(CCD) camera was used for studying surface structure. The contact potential difference (CPD) method was used for monitoring work function changes. The stored images of the LEED patterns were used to obtain the peak intensities and full widths at half maximum (FWHM) for the particular spots. The same molybdenum specimen as in our previous works ${ }^{3,14}$ was used as substrate, which is characterized by average (112) terrace sizes

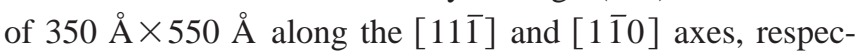
tively. An atomic-beam source of $\mathrm{Sr}$ was built by thermal activation in $\mathrm{UHV}$ of $\mathrm{SrO}_{2}$ mixed with $\mathrm{Al}$ powder. ${ }^{15} \mathrm{~A}$ base pressure in the vacuum chamber between 0.7 and 1.5 $\times 10^{-11}$ mbar was kept all the time, i.e., both during deposition and subsequent measurements.

The Mo(112) sample was routinely cleaned by many heating cycles in oxygen between 800 and $2000 \mathrm{~K}$. Oxygen was finally removed by several heating cycles to $2400 \mathrm{~K}$ in vacuum. Auger electron spectroscopy was used to check purity of the substrate and adsorbate. The coverage $\theta$ is defined as the ratio of adsorbate to substrate surface atom concentrations. It was determined from the deposition time and from the work function measurements calibrated by means of LEED in the coverage range $\theta=0-0.5 \mathrm{ML}$. In this range of coverage the relation between the coverage and the LEED pattern is unique.

Deposition of the adsorbate was carried out at the lowest available substrate temperature $(80 \mathrm{~K})$. Measurements were only started after annealing these layers to $700 \mathrm{~K}$. Each annealing step lasted $30 \mathrm{~s}$ and was followed by a gradual temperature decrease at a rate of $-4 \mathrm{~K} / \mathrm{s}$ back to the base temperature. Data were taken with a slow scan CCD camera at the intervals of $4 \mathrm{~s}$ during heating and cooling at a rate of 0.2

$\mathrm{K} / \mathrm{s}$ (between 400 and $600 \mathrm{~K}$ at $0.5 \mathrm{~K} / \mathrm{s}$ ). All the LEED data shown were taken at an electron beam energy of $80 \mathrm{eV}$.

\section{RESULTS AND DISCUSSION}

\section{A. Depinning of the $p(5 \times 1)$ structure}

After preparation of a layer with $0.20 \mathrm{ML}$ of $\mathrm{Sr}$ as described above, a $p(5 \times 1)$ pattern is seen. At the lowest temperature the superstructure spots are equally spaced, i.e., the structure is commensurate. When heating the sample, however, the separation of the superstructure spots starts to change, i.e., the structure undergoes a commensurateincommensurate phase transition. This transition is reversible, as explicitly checked by the heating and cooling cycles. Since the separation of the superstructure spots decreases, the average lattice constant of the incommensurate phase must increase by thermally excited formation of light domain walls in the $p(5 \times 1)$ structure.

Since the average $\mathrm{Sr}$ density on the surface is unchanged, the ordered incommensurate structure cannot accommodate the total coverage, for which many possibilities exist. We have carefully checked for any significant increase of background intensity at specific locations in $k$ space as a function of temperature, but could not find any. Therefore, the excess $\mathrm{Sr}$ coverage must be either accumulated in point defects, i.e., small islands that are not correlated with respect to each other. As another alternative, the excess $\mathrm{Sr}$ atoms could lead to doubling of $\mathrm{Sr}$ chains and thus contribute only to the integer-order beams. The fact that actually two transitions occur can be seen from the small anomalies found in the integrated intensities of superstructure beams [see Fig. 2(a)]. Integrated intensities have been determined by integrating in $k$ space over the squares that are twice as long in [11i] direction as in [1 $\overline{1} 0]$ direction, taking an adjacent area of equal size along the long sides of the integrated rectangle as background. The results obtained are insensitive to the actual size of integrated area, as also shown in this figure. They have not been corrected by a Debye-Waller factor. The anomalies are more clearly visible and quantifiable in the derivatives with respect to temperature, shown in Fig. 2(b). This derivative has been obtained by fitting second-order polynomials to 12 point sections of the curves of Fig. 2(a). Clearly two transitions can be identified. Taking the maximal slopes as parametrization of the transition temperatures, these transitions are at the temperatures of $96 \mathrm{~K}$ and $210 \mathrm{~K}$, respectively. Similar measurements have also been carried out for a coverage of $0.22 \mathrm{ML}$ with similar results. Only the transition temperatures shift to $89 \mathrm{~K}$ and $218 \mathrm{~K}$, respectively.

In order to get more quantitative information of the shifts of superstructure spots, we carried out fits with Gaussians to the profiles of line scans through integer and fractional order

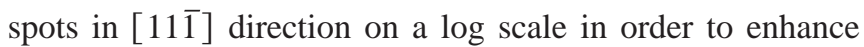
the weight of the superstructure beam intensity. The results of these fits for the spot separation are shown in Fig. 3, those for the full widths at half maximum in Fig. 4.

Obviously, the first anomaly seen in Fig. 2 coincides with the deviation of the lattice constant of the superstructure from commensurability, as seen from Fig. 3. Therefore, this 
a)

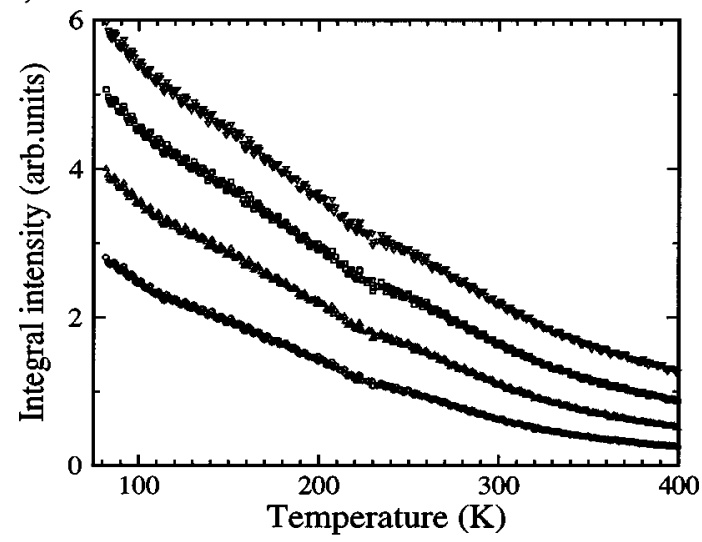

b)

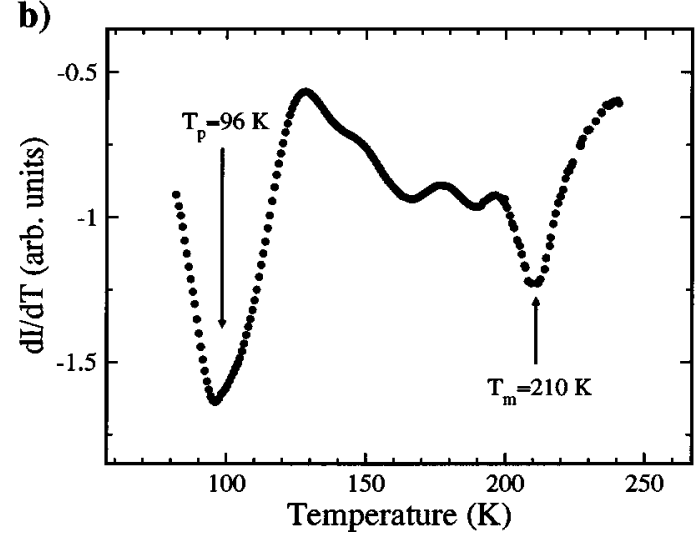

FIG. 2. Anomalies seen in integrated intensities of superstructure spots of the $p(5 \times 1)$ structure (a). As an example, the $(-0.2,1)$ spot is shown. The various curves (from bottom to top) are for integration over recangles (see text) with long sides of $3.2 \%$, $4.3 \%, 5.4 \%$, and $6.5 \%$ Brillouin zone(BZ), respectively. (b) Differentiated curve of the $3.2 \%$ data set. Electron energy $80 \mathrm{eV}$.

transition can be identified as a depinning transition into a striped soliton phase. ${ }^{12}$ The second transition at higher temperature must then correspond to melting of the soliton lattice consisting of domain walls. This second transition has obviously no direct influence on the average lattice constant of the incommensurate phase, since no anomaly is seen there. It is interesting to note, however, that the average lat-
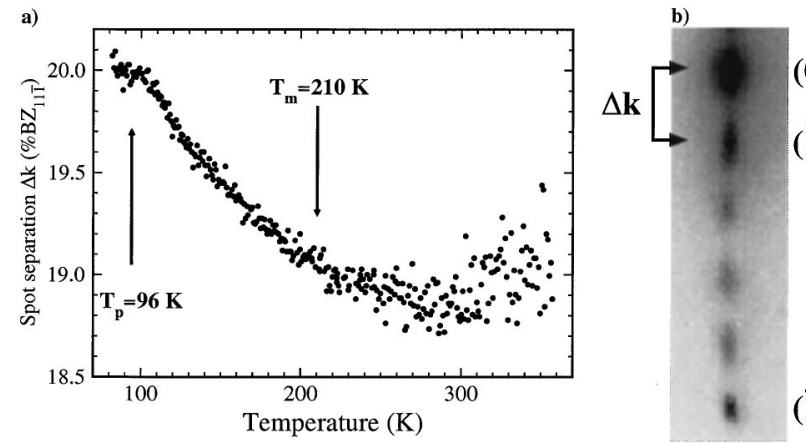

FIG. 3. Position of the ( $\left.\frac{\overline{4}}{5} 1\right)$ spot as a function of temperature. For clarity, a linear section of the corresponding LEED pattern on Fig. 1 is shown on the right. a)

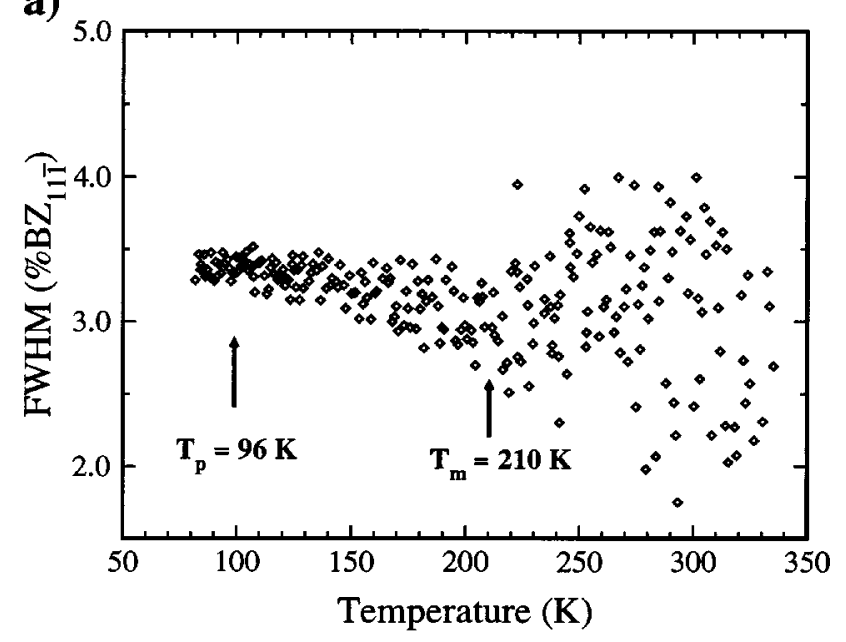

b)

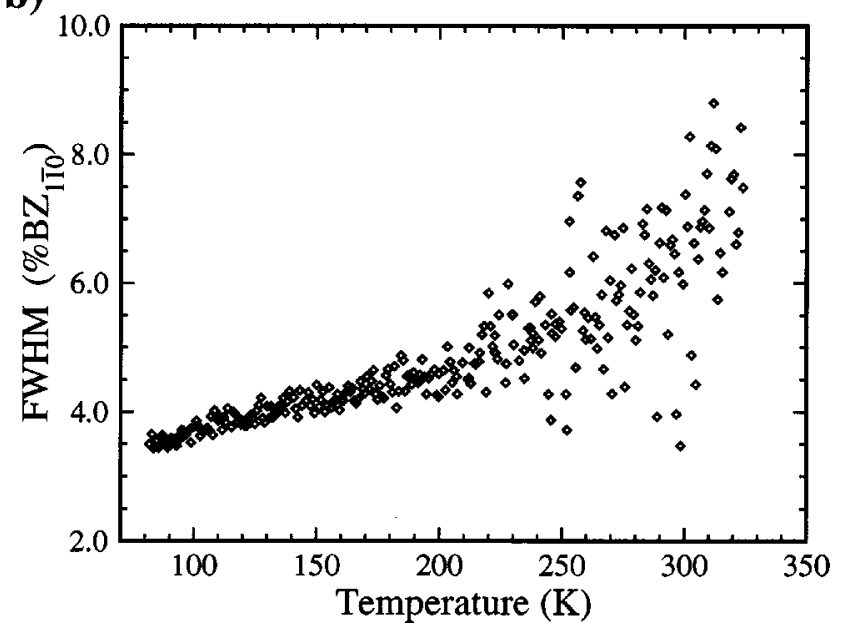

FIG. 4. (a) FWHM of the ( $\left.\frac{\overline{4}}{5} 1\right)$ spot in $[1 \overline{1} 0]$ direction. (b)

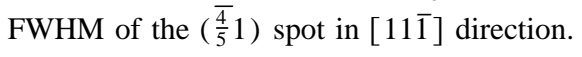

tice constant of the short-range order remaining in the fluid phase above $T_{m}$ goes back towards commensurability, although commensurability is not reached again.

The strong anisotropy in this system is also reflected in the FWHMs shown in Fig. 4. This figure shows two main features. The top part shows the half widths in the direction along the Sr chains. In this direction correlation lengths are not only higher than in the direction perpendicular to the chains, the FWHMs along the Sr chains are also essentially unaffected by the transitions. The FWHMs in this direction as a function of temperature are even slightly decreasing up to $T_{m}$. Above this temperature the scatter increases dramatically, but the average value does not change much. From this result we conclude that the $\mathrm{Sr}$ chains in the $p(5 \times 1)$ structure seem to be essentially rigid on the length scale of $30-50$ lattice constants in the temperature range investigated.

The situation is completely different in the direction perpendicular to the chains (see lower part of Fig. 4). Here the FWHM increases monotonically as a function of temperature once the depinning temperature has been exceeded, and the onset of $2 d$ melting is coupled with a sudden change of 

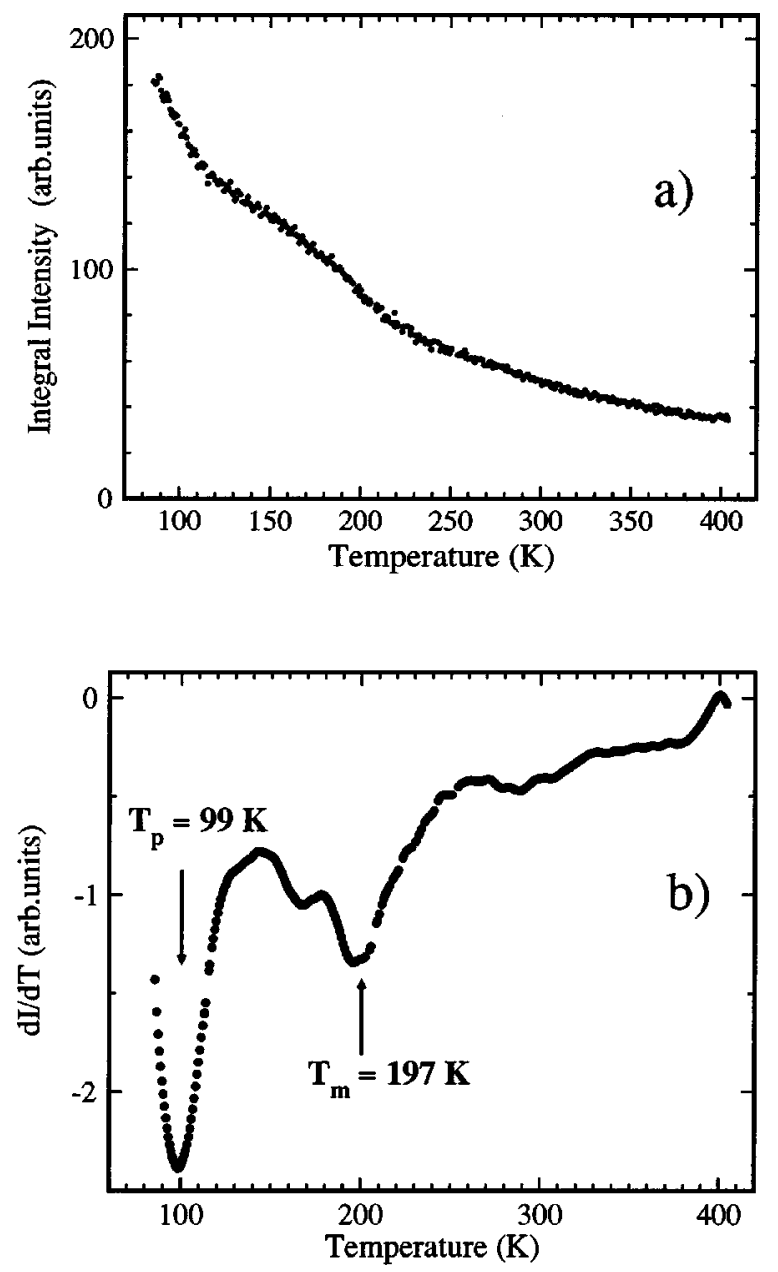

FIG. 5. Integrated intensities for the $p(8 \times 1)$ structure of $\mathrm{SrMo}(112)$ for a coverage of $0.11 \mathrm{ML}$ Sr. In (a) the integrated intensity of the $\left(\frac{\overline{1}}{8} 1\right)$ spot is shown for a rectangular integration area of $4.3 \% \mathrm{BZ}_{(11 \overline{1})}$ (for details, see text). Electron beam energy $80 \mathrm{eV}$. (b) Differentiated curve of (a).

slope. This shows that the domain wall lattice is far from being perfect. Since the correlation lengths are comparatively short, it is an open question whether the gradual increase of FWHM in this phase can be taken as indication for algebraic decay of long-range order. Even far above the melting transition there is considerable correlation still detectable. Similar observations have been made for the Li/ Mo(112) system. ${ }^{14}$

\section{B. Depinning of the $p(8 \times 1)$ structure}

Very similar observations have been made for the $p(8$ $\times 1)$ structure. In analogy to the $p(5 \times 1)$ results, we show in

Fig. 5 integrated intensities of the $\left(\frac{\overline{1}}{8} 1\right)$ spot and their derivatives with respect to temperature for a Sr coverage of 0.11 ML. Similar to the $p(5 \times 1)$ structure, two anomalies are found at temperatures of $99 \mathrm{~K}$ and $197 \mathrm{~K}$, respectively, which again can be identified with depinning and melting transitions.

The positions of the superstructure spots and their separation from the integer order beams have also been determined.
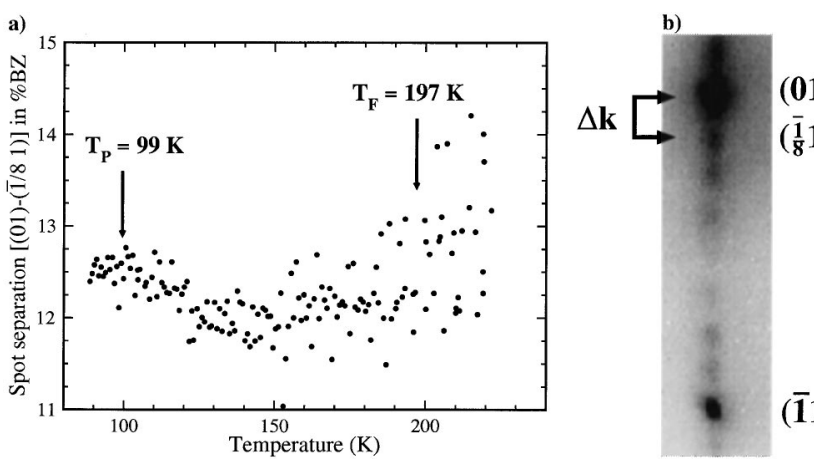

FIG. 6. Position of the $\left(\frac{\overline{1}}{8} 1\right)$-superstructure spot as a function of temperature. On the right side again the measured difference in $\Delta k$ is shown. The series of diffraction spots runs in (111) direction.

An example is shown in Fig. 6. Since the intensities of the superstructure spots of the $p(8 \times 1)$ structure are considerably lower than those of the $p(5 \times 1)$ structure, the scatter of the data is higher. The depinning transition, however, can also be identified in this graph. Again, the unit cells increase in size in the incommensurate phase. The size of the observed shift and the trend to go back to an average commensurate unit cell is also found here.

These results demonstrate that both chain systems originate from the same type of lateral interactions so that they undergo the same type of phase transitions. Even the observed transition temperatures are very close so that similar magnitudes of lateral interactions can be concluded for both structures.

\section{Depinning by thermal excitation of domain walls}

As already mentioned in the Introduction, the most remarkable property of the system investigated here, and presumably of the whole class of similar systems, is the the ordered arrangment of simple atomic chains at large distances of five and eight lattice constants. These correlations prevail as short-range order even up to room temperature. In addition to this remarkably strong interaction over such large distances, it is also important to note that only distances of five and eight lattice constants seem to be favorable, whereas the others are obviously unstable at low temperatures. These other distances, however, may be accessible as thermally excited states, which finally lead to disorder of the whole system. Apart from the lateral interactions in this system, which are responsible for ordering, the strong chemical bond of $\mathrm{Sr}$ to the Mo substrate leads to bonding of $\mathrm{Sr}$ on well-defined adsorption sites ${ }^{4}$ and to an adsorption potential that is much stronger than the lateral interactions. Domain walls can still be formed in such systems, but in contrast to physisorbed systems they have to be atomically sharp since they occur only between discrete lattice sites. In this section we show that depinning observed in the system studied here is fully compatible with the oscillatory type of interaction caused by charge density waves.

If we assume that the coupling between chains indeed happens by a combination of dipolar repulsion and an oscillatory contribution from the adsorbate-induced Friedel oscil- 


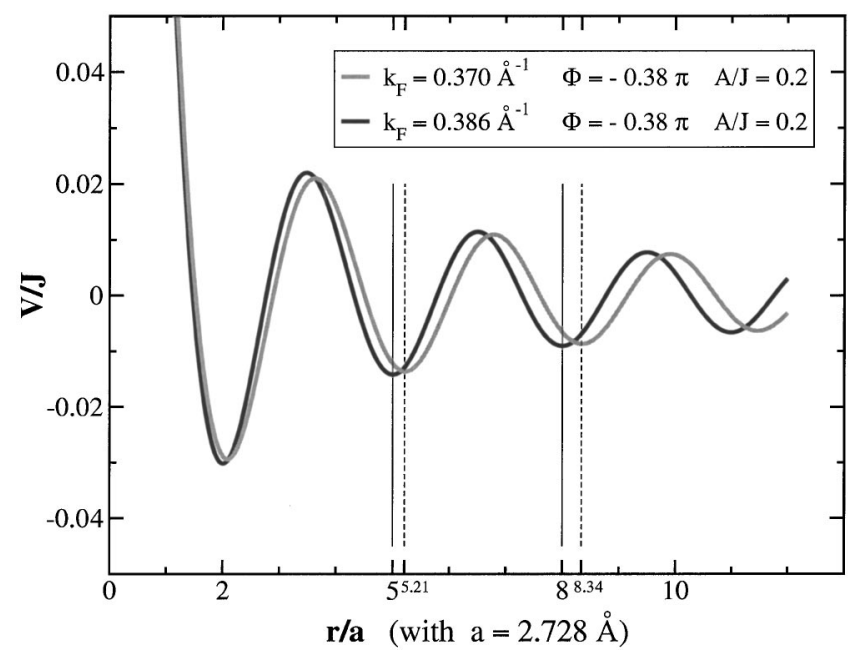

FIG. 7. Dependence of the two-particle interaction on distance assuming contributions from the dipolar repulsion and from the Friedel oscillations for two values of $k_{F}$, which demonstrate the sensitivity to the value of $k_{F}$.

lations, the interaction between the chains is essentially one dimensional and the oscillatory part is of fairly long range, since it decays only as $l^{-1}$ (Ref. 16). Therefore, it takes the following asymptotic form for distances $l \gg a$ :

$$
V=\frac{J}{l^{3}}+\frac{A}{l} \cos 2 k_{F}+\phi,
$$

which is plotted in Fig. 7. The ratio $A / F=0.2$ used here stems from an optimization ${ }^{17,18}$ to describe and simulate the stable phases of this system in the framework of lattice gas models and can be taken as a first approach to the real value. $k_{F}$ in this figure was chosen so that minima appear both at the distances of five and eight lattice constants. The second curve is only drawn to demonstrate the sensitivity to changes in $k_{F}$.

If we use $k_{F}=0.386 \AA^{-1}$ and $\Phi=0.38 \pi$ (see Fig. 7) and sum up the contributions of many chains at a distance of five lattice constants (leaving out that by which the potential should be measured), we obtain the effective potential shown as upper graph in Fig. 8. It produces a clear minimum at a distance of five lattice constants. Occupation of the two other (even deeper) minima at the distances of approximately $2 a$ and $8 a$ seems to be even more favorable, but a $p(2 \times 1)$ structure has not been seen for the clean $\mathrm{Sr} / \mathrm{Mo}(112)$ system. This means that at $2 a$ we are below the asymptotic limit of Eq. (1), and more direct interactions dominate. It is worth noting, however, that the lateral relaxations in the substrate found with $\operatorname{LEED}^{4,5}$ still show an increased atom density at these distances.

As mentioned above, domain walls in this system can only be created by discrete changes of separations between Sr chains by one or more lattice units of the substrate because of the strong site specific bonding of Sr. The activation energy necessary to create domain walls on the discrete lattice of substrate sites was simulated by introducing onedimensional stacking faults into the periodic Sr chain system.
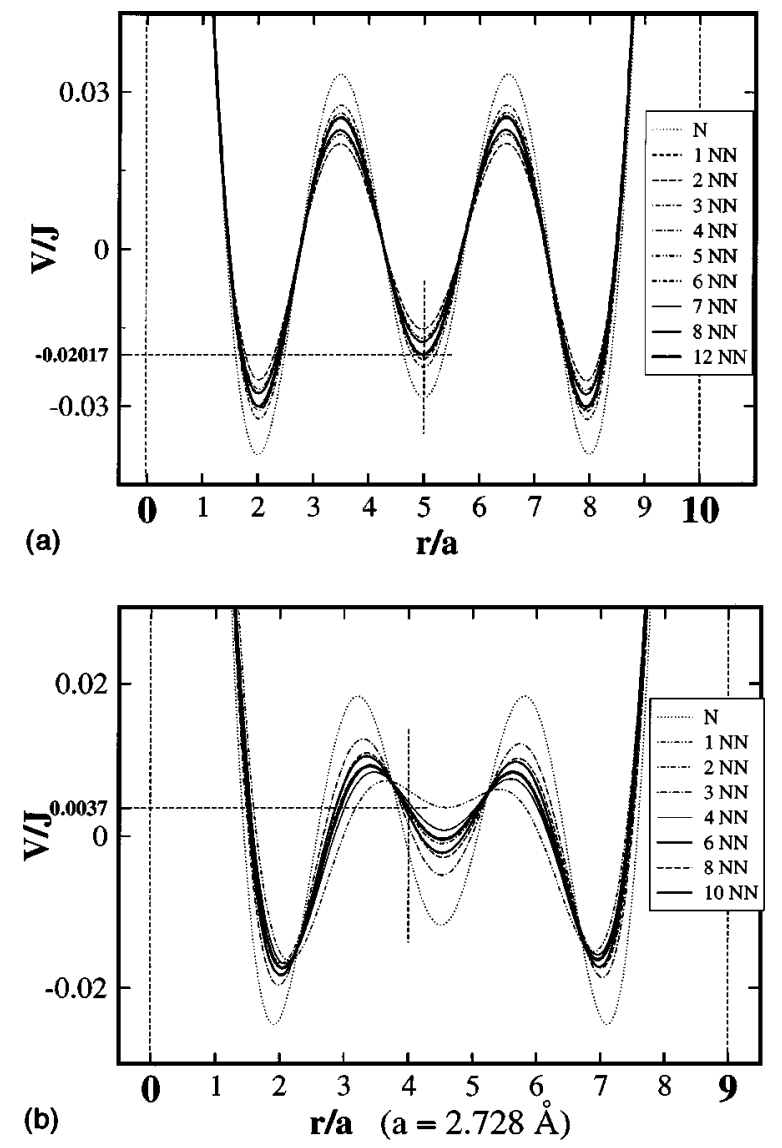

FIG. 8. Top: Effective potential for a $\mathrm{Sr}$ chain interacting with $n=1,2,3,4, \ldots, 20$ neighboring chains located at distances of multiples of $5 a$. The same parameters as in Fig. 7 are used $\left(k_{F}\right.$ $=0.386 \AA^{-1}$ ). Bottom: same as top, but with a stacking fault of $-1 a$. $N$ means neighbor, NN means nearest neighbor.

As an example, we show in the lower part of Fig. 8 the resulting potential energy curve for a heavy domain wall, where the separation between two chains is reduced by one lattice constant only at one point. As seen there, a new but shallower minimum now appears at $4.5 a$. Sr chains, however, can only be located at separations close to integer multiples of $a$. This increases the necessary activation energy further. An analogous increase of energy is found for light

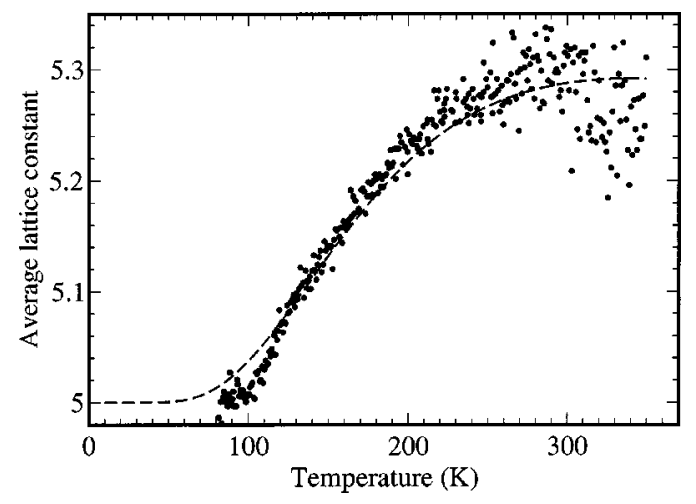

FIG. 9. Fit to the measurements of the average shift of the chain separation as a function of temperature. 
domain walls, which correspond to chain separations of 6,7, etc., lattice constants. Keeping all other parameters constant, the necessary activation energy of a light wall with $6 a$ separation is a factor of 2 lower than that of the heavy wall with $4 a$ separation. This asymmetry of activation energies is a necessary precondition for the continuous shift of the average lattice constant as a function of temperature.

Indeed, the observed continuous shift of the chain separation, as deduced from the LEED experiments as a function of temperature can be simulated by assuming a still low con- centration of domain walls and thermal equilibrium between the various types of domain walls. Since we have only imcomplete knowledge of the equation of state of the surface layer, we restrict ourselves to a consideration of the energetics only, i.e., we neglect any entropic differences between the various types of domain walls. In order to get an estimate of the energies of activation for the domain walls, we assume as a first approximation that only wall separations of 3, 4, 6, and $7 a$ occur. With this assumption we carried out a fit of the average lattice constant of the chain system by fitting the function

$$
\bar{d}=\frac{3 e^{-\left(V_{3}-V_{5}\right) / k_{B} T}+4 e^{-\left(V_{4}-V_{5}\right) / k_{B} T}+5+6 e^{-\left(V_{6}-V_{5}\right) / k_{B} T}+7 e^{-\left(V_{7}-V_{5}\right) / k_{B} T}}{e^{-\left(V_{3}-V_{5}\right) / k_{B} T}+e^{-\left(V_{4}-V_{5}\right) / k_{B} T}+1+e^{-\left(V_{6}-V_{5}\right) / k_{B} T}+e^{-\left(V_{7}-V_{5}\right) / k_{B} T}}
$$

to the data. A comparison of this fit to the experimental data is shown in Fig. 9. Apart from the critical region, where entropic contributions can of course not be neglected, we obtain a reasonably good fit. As expected from the above model, the values of the parameters fall into two groups for light and heavy walls. Although the activation energy of the heavy walls was found to be close to $65 \mathrm{meV}$ per $\mathrm{Sr}$ atom in the chain, that of the light walls tested was around $38 \mathrm{meV}$. The ratio is close to that estimated from our model [Eq. (1)] for activation of light and heavy walls, where a factor of 2.5 was obtained. Since our intention here is only to clarify the basic mechanism and to give reasonable estimates for the interaction energies, no further refinement of parameters was attempted. This results in effective interaction constants $J$ and $A$ [see Eq. (1)] of approximately $3 \mathrm{eV} \AA^{3}$ and $0.6 \mathrm{eV} \AA$, respectively.

\section{CONCLUSIONS}

We have shown in this analysis that interactions between chains of adsorbed species on anisotropic metal substrates can be extremely long range. Only very few coupling mechanisms decay sufficiently slowly to be effective here, and the coupling via an adsorbate-induced charge density wave is one of them.

In fact, our experimental data seem to be fully consistent with this coupling mechanism for two reasons.

(1) The lateral relaxations investigated previously ${ }^{4,5}$ directly suggest this mechanism to be effective.
(2) The mechanism of coupling between adsorbate chains by the adsorbate-induced Friedel oscillations should be strongly sensitive to the changes of electron density in the surface states involved in this mechanism and/or to modifications of these states themselves.

This can easily be achieved by adding, e.g., electronegative species to these layers of electropositive $\mathrm{Sr}$ atoms. Indeed such modifications have been observed by doping these layers with concentrations of oxygen much less than one atom per unit cell. ${ }^{19}$ This corroborates the model for the effective chain interactions qualitatively. A detailed description of these modifications for the structures discussed here will be presented elsewhere. ${ }^{20}$

The phase transitions seen in this study, depinning and finally melting of the domain wall lattice, are characteristic for those expected in strongly anisotropic two-dimensional systems with large unit cells and agree with the behavior expected from the theory. ${ }^{12}$ Although the coupling mechanism by adsorbate-induced Friedel oscillation of the electron density at the Fermi surface is quite evident, and although there are some similarities with the behavior at a Peierls transition such as the weak anomalies, ${ }^{21}$ it is a still open question whether such a transition actually occurs in the systems studied here.

\section{ACKNOWLEDGMENTS}

This work was supported by the Deutsche Forschungsgemeinschaft. We thank I. Lyuksyutov for stimulating discussions.
${ }^{\star}$ Electronic address: pfnuer@fkp.uni-hannover.de

${ }^{1}$ O. Braun and V. Medvedev, Sov. Phys. Usp. 32, 328 (1989).

${ }^{2}$ D. Kolthoff, H. Pfnür, A.G. Fedorus, and V. Koval, Surf. Sci. 439, 234 (1999).

${ }^{3}$ A. Fedorus, G. Godzik, V. Koval, A. Naumovets, and H. Pfnür, Surf. Sci. 460, 229 (2000).

${ }^{4}$ D. Kolthoff and H. Pfnür, Surf. Sci. 457, 134 (2000).

${ }^{5}$ D. Kolthoff and H. Pfnür, Surf. Sci. 459, 265 (2000).
${ }^{6}$ I. Yakovkin, Surf. Sci. 389, 48 (1997).

${ }^{7}$ G. Grüner, Density Waves in Solids (Addison-Wesley, Redwood City, 1994).

${ }^{8}$ T. Kidd, M. Chou, and T. Chiang, Phys. Rev. Lett. 85, 3684 (2000).

${ }^{9}$ K. Swamy, C. Deisl, A. Menzel, R. Beer, S. Penner, and E. Bertel, Phys. Rev. B 65, 121404 (2002).

${ }^{10}$ S. Lee, J. Ahn, N. Kim, C. Hwang, J. Chung, H. Yeom, S. Ryikov, 
and S. Hasegawa, Phys. Rev. Lett. 88, 196401 (2002).

${ }^{11}$ H. Yeom, S. Takeda, E. Rotenberg, I. Matsuda, K. Horikoshi, J. Schaefer, C. Lee, S. Kevan, T. Ohta, and T. Nagao et al. Phys. Rev. Lett. 82, 4898 (2001).

${ }^{12}$ I. Lyuksyutov, A. Naumovets, and V. Pokrovsky, TwoDimensional Crystals (Academic Press, Boston, 1992).

${ }^{13}$ D. Jürgens, G. Held, and H. Pfnür, Surf. Sci. 303, 77 (1994).

${ }^{14}$ A. Fedorus, D. Kolthoff, V. Koval, I. Lyuksyuotov, A. Naumovets, and H. Pfnür, Phys. Rev. B 62, 2852 (2000).

${ }^{15}$ V. Gavriluk and V. Medvedev, Sov. Phys. Solid State 8, 1439 (1966).
${ }^{16}$ B. Gumhalter and W. Brenig, Surf. Sci. 336, 326 (1995).

${ }^{17}$ F. Bagehorn, J. Lorenc, and C. Oleksy, Surf. Sci. 349, 165 (1996).

${ }^{18}$ C. Oleksy and J. Lorenc, Phys. Rev. B 54, 5955 (1996).

${ }^{19}$ G. Godzik, H. Pfnür, and I. Lyuksyutov, Europhys. Lett. 56, 67 (2001).

${ }^{20} \mathrm{G}$. Godzik and H. Pfnür (unpublished).

${ }^{21}$ J. W. Brill, in Physics and Chemistry of Low-Dimensional Inorganic Conductors, edited by C. Schlenker, J. Dumas, M. Greeblatt, and S. van Salen (Plenum, New York, 1996), Vol. 354 of NATO ASI Series B, pp. 345-355. 